\title{
Photoconductivity of ZnTe thin films at elevated temperatures ${ }^{\dagger}$
}

\author{
N MAZUMDAR, R SARMA, B K SARMA and H L DAS* \\ Department of Physics, Gauhati University, Guwahati 781 014, India
}

MS received 1 May 2004; revised 10 January 2006

\begin{abstract}
Photoconductivity of thermally evaporated $\mathrm{ZnTe}$ thin films was studied at different elevated temperatures. A gap type cell configuration with Al electrodes on glass substrates was used. The conductivity was found to obey two distinct conduction mechanisms within the region of applied fields. At low fields the photoconduction is ohmic and at high fields it is of Poole-Frenkel type. With increase of ambient temperatures, the Poole-Frenkel conductivity regions were found to extend to lower fields. The temperature dependence of dark conductivity also was found to be of similar nature.
\end{abstract}

Keywords. Photoconductivity; ZnTe thin films; Poole-Frenkel.

\section{Introduction}

Zinc telluride is one of the important semiconductor materials of II-VI group because of its extensive potential applications in different opto-electronic devices (Weast 1977-78; Kalita et al 1998). ZnTe thin films grown at room temperature and higher substrate temperatures were found to be polycrystalline in nature. These films have large number of grain boundary potentials as well as other native defects. At room temperature and higher ambient temperatures the potential barrier localized in the grain boundaries are modulated by the externally applied fields. Hence there is a possibility in these films that the conductivity is modified by externally applied fields. Such field dependent conductivity is called the Poole-Frenkel conductivity (Simmons 1970). Reports on the electrical conduction in thin $\mathrm{ZnTe}$ films (Shirakawa et al 1970; Holt and Mufti 1973) often consider the form of one $I-V$ curve only. However, this can be a misleading information on which to judge the conduction mechanism; an investigation of the temperature dependence of conductivity in such films is also necessary (Burgelman 1980). Recently, some studies on Frenkel pairs of two sublattices of ZnTe (Ufting et al 2000) and on the predominance of Poole-Frenkel mechanism in the photoconductivities of $\mathrm{ZnTe}$ thin films at room temperature (Sarma et al 2001) have also been reported. In this paper, an attempt has been made to assess the predominance of the Poole-Frenkel conduction mechanism in the dark and photoconductivities of ZnTe thin films at room temperature and higher ambient temperatures.

\footnotetext{
*Author for correspondence (hld_guphys@ rediffmail.com) ${ }^{\dagger}$ The paper was presented at the 6th Asian Thermophysical Properties Conference (6th ATPC), held at Gauhati University, during 8-11 October 2001.
}

\section{Experimental}

ZnTe thin films of different thicknesses were deposited on properly cleaned glass substrates with the help of a Hind High Vacuum Coating unit (HINDHIVAC 12A4) at the vacuum, $10^{-6}$ torr. Pure (99.999\%) $\mathrm{ZnTe}$ in coarse powder form obtained from Koch Light Lab. UK, was used as the source material. Prior to deposition of the thin films, high purity aluminium electrodes were vacuum evaporated on the properly cleaned glass substrates on which the films were then deposited to obtain gap type cells of $7 \mathrm{~mm}$ gap. Thin tantalum boats were used as source heater. The area of thin film specimens between the electrodes is $7 \times 10 \mathrm{~mm}$. The thickness of the deposited films were measured with the help of a multiple beam interferometer with an accuracy of $\pm 15 \AA$. During the time of observation the sample was placed inside an evacuated glass jacket with the help of a suitable sample holder being suspended by thin enamelled copper wires.

A suitable optical arrangement was made to illuminate the sample uniformly in which a tungsten halogen lamp (250 watt) with an attached parabolic focussing mirror was used. Light intensity was measured with the help of a sensitive APLAB luxmeter. High ambient temperatures were achieved by using a heater connected to a stabilized power supply and temperatures were measured with the help of a copper constantan thermocouple and a digital microvoltmeter arrangement. Photocurrent and dark currents were measured with the help of an ECIL electrometer amplifier of input impedance $10^{14} \mathrm{ohm}$ and higher. A series of highly stable dry cells each of emf $9 \mathrm{~V}$ was used to provide applied bias. The entire experimental set up (including the observer) was housed in a suitably fabricated Faraday cage to avoid the pick up noise. 


\section{Results and discussion}

We define the photocurrent density, $J_{\mathrm{ph}}$, by $J_{\mathrm{L}}-J_{\mathrm{D}}=J_{\mathrm{ph}}$, where $J_{\mathrm{L}}$ and $J_{\mathrm{D}}$ are the light and dark current densities, respectively. The $J_{\mathrm{ph}}$ vs $V_{\mathrm{a}}$ characteristics, drawn corresponding to room temperature and higher ambient temperatures in the applied bias $\left(V_{\mathrm{a}}\right)$ range from 0 to $+108 \mathrm{~V}$ and from 0 to $-108 \mathrm{~V}$ are shown in figures 1 and 2, respec-

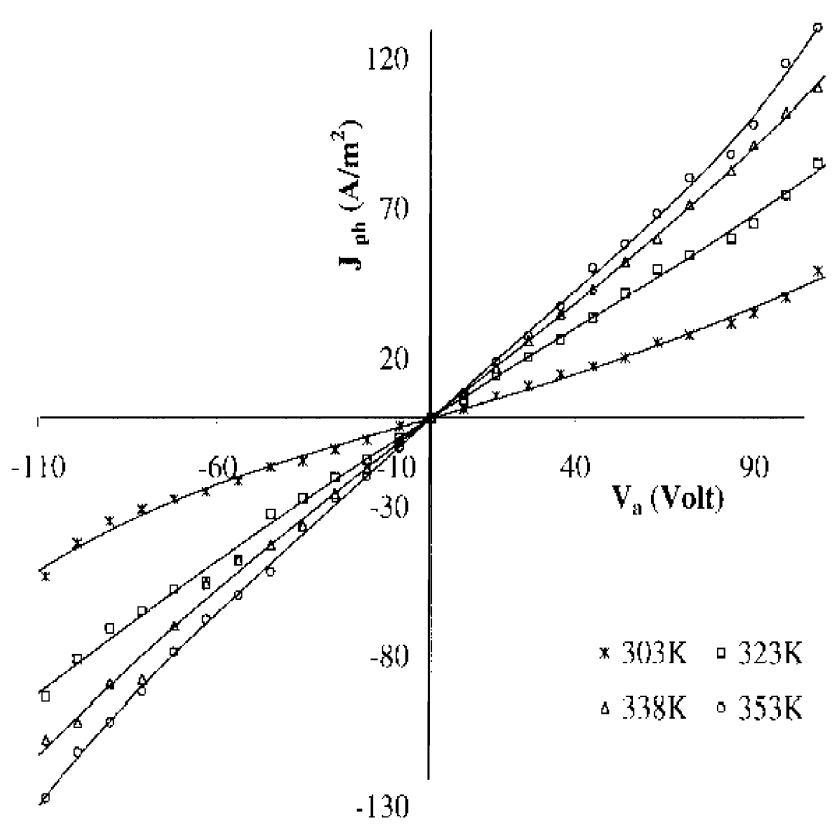

Figure 1. Photocurrent density $\left(J_{\mathrm{ph}}\right)$ vs applied bias $\left(V_{\mathrm{a}}\right)$ for ZnTe thin films grown at $303 \mathrm{~K}$.

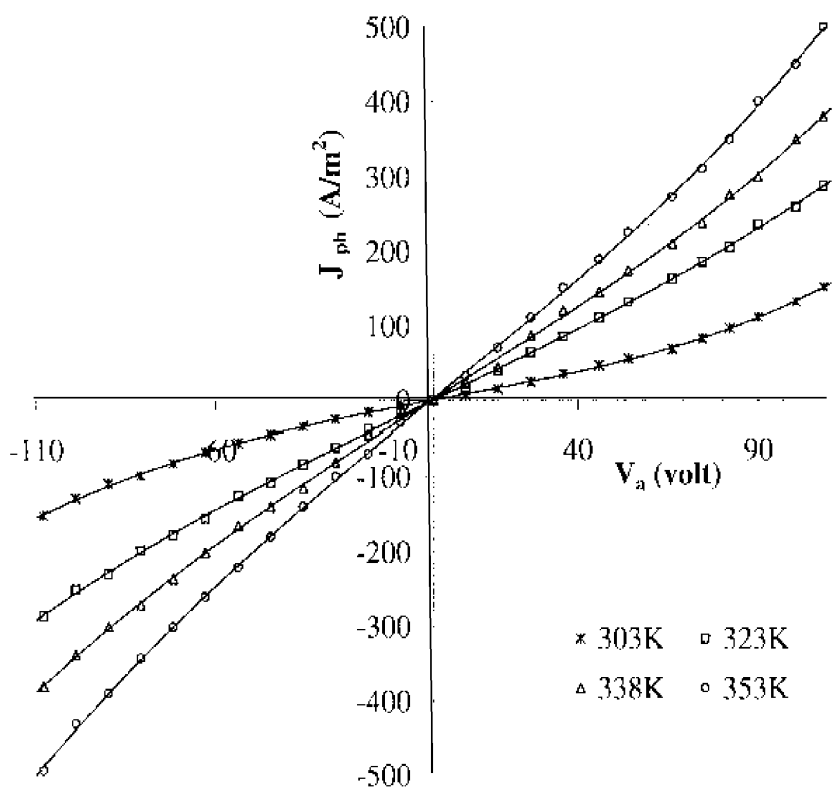

Figure 2. Photocurrent density $\left(J_{\mathrm{ph}}\right)$ vs applied bias $\left(V_{\mathrm{a}}\right)$ for ZnTe thin films grown at $403 \mathrm{~K}$. tively for a particular intensity of white light (59830 lux fixed). The $J_{\mathrm{ph}}-V_{\mathrm{a}}$ curves are found to obey two distinct conduction mechanisms in $\mathrm{ZnTe}$ thin films grown at room temperature $(303 \mathrm{~K})$ and higher temperature substrates (403 K). In figures 3 and 4 , the curve of $\ln J_{\mathrm{ph}}$ vs $F^{1 / 2}$ have been drawn. Here $F$ is the applied field.

\subsection{Variation of photocurrent density at low fields}

There is a low voltage range (both for +ve and -ve bias) in which the $J_{\mathrm{ph}}$ vs $V_{\mathrm{a}}$ characteristics are linear and pass

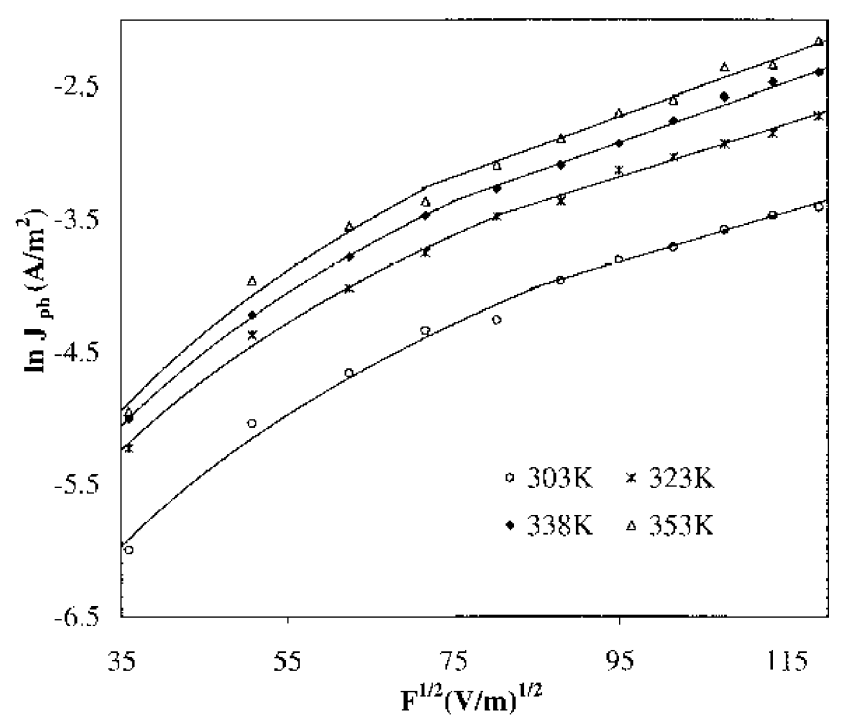

Figure 3. $\ln J_{\mathrm{ph}} \mathrm{vs} F^{1 / 2}$ of $\mathrm{ZnTe}$ thin films grown at $303 \mathrm{~K}$.

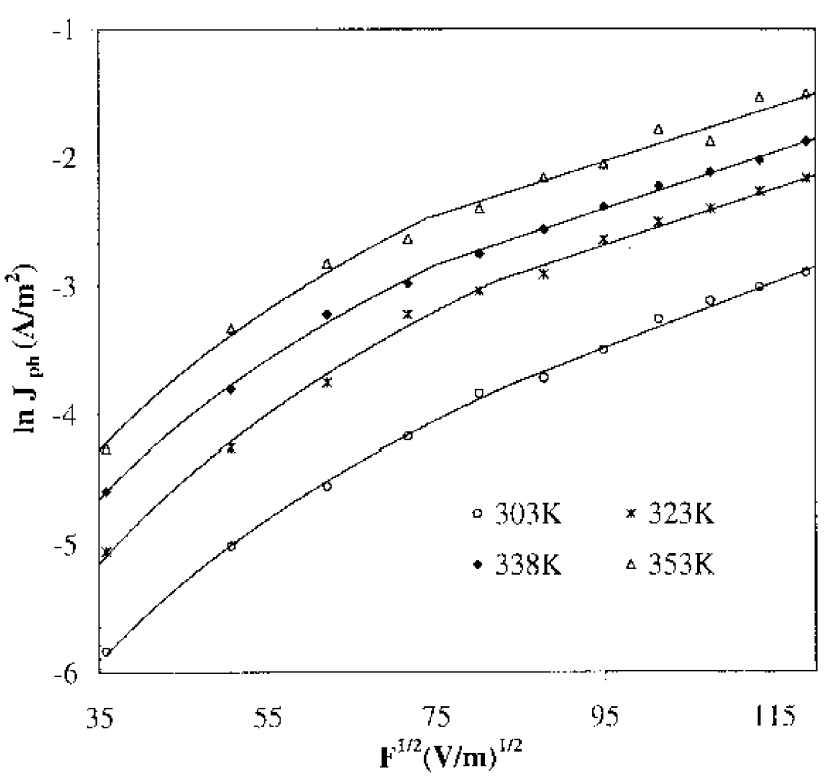

Figure 4. $\ln J_{\mathrm{ph}} \mathrm{vs} F^{1 / 2}$ for ZnTe thin films grown at $403 \mathrm{~K}$. 
symmetrically through the origin for each temperature for both the growing conditions. This shows that the photocurrent and applied voltage relationship is ohmic in this voltage range. It is also observed that the range of applied bias for this linear region decreases for both polarities as the ambient temperature increases (from 303-363 K) for a fixed intensity of light. This nature is similar for different intensities of light. This also implies that in the studied ZnTe thin films vacuum evaporated $\mathrm{Al}$ electrodes make ohmic contact with the thin films in the low field regions.

\subsection{Variation of photocurrent density at high fields}

Beyond the low voltage region, the $J_{\mathrm{ph}}$ vs $V_{\mathrm{a}}$ curves become non-linear. It may be noted that the linear dependence region extends to lower fields as the ambient temperature increases, for fixed intensity of light. In the respective high field regions, the $\ln J_{\mathrm{ph}}$ vs $F^{1 / 2}$ graphs are linear for each ambient temperature and fixed intensity of light for both the films grown at room temperature substrates and higher temperature substrates as shown in figures 3 and 4 . This clearly indicates that in the high field region the conduction mechanism is of Poole-Frenkel type. The current due to Poole-Frenkel type of conduction mechanism is given by

$$
J=J_{0} \exp \left(\beta_{\mathrm{PF}} F^{1 / 2} / k T\right)
$$

where $J_{0}(=\sigma F)$ is the low field current density, $\beta_{\mathrm{PF}}$ the Poole-Frenkel coefficient and other symbols have their usual significance. The nature of the two conductivity mechanisms are found to be the same for both room temperature and higher substrate temperature films. Obviously it can be mentioned that the Poole-Frenkel region increases as the ambient temperature increases.

A part of the thermally excited carriers due to heating together with the photogenerated carriers due to illumination goes to neutralize some fraction of the corresponding localized charges in the depletion regions in the grain boundary potential barriers. As a result of these two factors, the grain boundary potential $\left(\phi_{\mathrm{b}}\right)$ is reduced. This in turn enhances the effective mobility given by

$$
\mu^{*}=\mu_{0} \exp \left(-q \phi_{\mathrm{b}} / k T_{0}\right)
$$

where $T_{0}$ is a characteristic temperature of the $\mathrm{ZnTe}$ thin films and $\mu_{0}$ the mobility of the carriers with no barrier effect. Clearly this introduces an additional exponential factor in $J_{\mathrm{ph}}$. Hence the linear portion of $\ln J_{\mathrm{ph}}$ vs $F^{1 / 2}$ plots increases with thermal activation at constant illumination and photoexcitation at ambient temperatures. From the plots of figure 4, it is also clear that the Poole-Frenkel conductivity process becomes more prominent in the studied $\mathrm{ZnTe}$ thin films deposited at higher substrate temperature. As these films are highly photosensitive, a part of the ther- mally excited carriers together with the larger number of photogenerated carriers also neutralize some fraction of the localized charges in the depletion regions in the grain boundary potential barriers. Because of this process, conductivity is found to increase exponentially with the applied field. Similar types of characteristics have also been observed for both types of the films for dark current also.

\subsection{Calculation of Poole-Frenkel coefficient}

From the linear portion of figures 3 and 4, the PooleFrenkel coefficients have been calculated. From the $J_{\mathrm{ph}}$ vs $F^{1 / 2}$ plots, slopes have been calculated from the linear regions, given by $m=\beta_{\mathrm{PF}} / k T$. Hence Poole-Frenkel coefficient is $\beta_{\mathrm{PF}}=m k T$. The Poole-Frenkel coefficients for different ambient temperatures for both types of the films grown at $303 \mathrm{~K}$ and $403 \mathrm{~K}$ substrate temperatures have been calculated. $\beta_{\mathrm{PF}}$ have been found to lie in the range from $6.533 \times 10^{-4} \mathrm{eV} \mathrm{m}^{1 / 2} \mathrm{~V}^{-1 / 2}$ to $10.656 \times 10^{-4} \mathrm{eV} \mathrm{m}^{1 / 2}$ $\mathrm{V}^{-1 / 2}$ for films of room temperature substrates and from $5.226 \times 10^{-4} \mathrm{eV} \mathrm{m}^{1 / 2} \mathrm{~V}^{-1 / 2}$ to $6.972 \times 10^{-4} \mathrm{eV} \mathrm{m}^{1 / 2} \mathrm{~V}^{-1 / 2}$ for films of higher temperature substrates. Within the ambient temperature range 303-353 K, the Poole-Frenkel coefficient decreases as the ambient temperature increases. It is also observed that the values of $\beta_{\mathrm{PF}}$ for films grown at room temperature substrates is marginally higher than those for films grown at higher temperature substrates. The PooleFrenkel coefficient for dark is nearly same as in the case of photocurrent.

\section{Conclusions}

It has been observed that in thermally deposited polycrystalline ZnTe thin films, the photocurrent density characteristics have two distinct conductivity regions within the presently studied applied bias range. From this study it can be concluded that the photocurrent density increases linearly and exponentially in low and high applied bias regions, respectively. In high applied bias region the exponential nature of photocurrent density increases with ambient temperature. From these studies, it is also clear that there is a decrease in the values of Poole-Frenkel coefficients from the observations taken in different ambient temperatures which are higher than the room temperature. These observations clearly support the process of neutralization of localized charges in the depletion regions in the grain boundary potential barriers due to the presence of both thermally generated carriers and photogenerated carriers in the studied ZnTe thin film samples.

\section{Acknowledgement}

We express our gratefulness to the Gauhati University Computer Centre, for providing us the necessary facilities for the computer work. 


\section{References}

Burgelman M 1980 Thin Solid Films 701

Holt D and Mufti A 1973 Solid State Electron. 161213

Kalita P C, Sarma K C and Das H L 1998 J. Assam Sci. Soc. 39 117

Sarma R, Mazumdar N and Das H L 2001 J. Assam Sci. Soc. (in press)
Simmons J G 1970 in Hand book of thin film technology (eds) L I Maissel and R Glang, Ch. 14, p. 25

Shirakawa T, Hayashi A and Nakai J 1970 J. Appl. Phys. 9 420

Ufting S J, Bozdog C, Linde M, Dömen A and Walkins G D 2000 Phys. Rev. B62 7195

Weast Robert C 1977-78 CRC hand book of chemistry and physics (Cleveland, Ohio: CRC Press, Inc.) 58th ed., p. 101 